\title{
Problems of integration of a manufacturing system with the business area of a company on the example of the Integrated Manufacturing Systems Laboratory
}

\author{
Grzegorz Ćwikła ${ }^{1, *}$ and Krzysztof Foit ${ }^{1}$
}

${ }^{1}$ Silesian University of Technology, Faculty of Mechanical Engineering, Institute of Engineering Processes Automation and Integrated Manufacturing Systems, Konarskiego 18A, PL44-100, Poland

\begin{abstract}
The paper presents issues related to data acquisition and communication between the manufacturing system and the business area of a company, understood as the transmission of real-time information about the status of the production system (the state of equipment, production tasks, workers, the flow of materials, work in progress and products), and, production orders, which is the basis of the Industry 4.0 concept. Data can be acquired from various sensors, PLCs and automatic identification systems, through interfaces (e.g. IO-Link, OPC, MTConnect), providing translation of data between different producer's equipment and software. The article presents assumptions, concept, interfaces, as well as hardware and software equipment of the Integrated Manufacturing Systems Laboratory, allowing research and teaching of integration of production and business areas. Laboratory workstations equipped with PLCs (3 different producers), sensors (e.g. distance, proximity, colour, position), actuators, HMI panels, hardware interfaces, RFID readers, barcode readers and vision systems, interfacing through OPC, IO-Link or Profinet with SCADA and other IT systems have been presented. An example of systems integration has also been presented.
\end{abstract}

\section{Introduction}

Issues related with the smooth flow of information in the enterprise are particularly important in the contemporary market situation, when companies are forced to compete with often global-scale competitors. Companies are looking for more sophisticated opportunities to optimize production and business processes, because the industry is running out of easy ways to increase efficiency by reducing operating costs, therefore it is necessary to ensure the continuous optimization of the functioning of the company, based on collecting and processing information about its functioning in real time, which is sometimes defined as an Industry 4.0 concept/initiative or Smart Manufacturing [1].

* Corresponding author: grzegorz.cwikla@polsl.pl 
Efficient, real-time feed of information about the dynamically changing state of machinery and equipment, production orders, flow of materials and semi-finished products, tasks performed by employees, quality of products and processes, and other necessary data can allow for the making of proper, optimised decisions, regarding i.a. dispatching of production tasks, improved use of available material, hardware (machines, equipment) and human resources, planning of equipment maintenance and investment in new machines [2].

The issue of acquisition of data from production systems is an interdisciplinary problem, requiring a combination of experience in areas such as industrial automation, computer science, electronics, communication and management. Industrial data acquisition for the needs of company management support is a broad issue, requiring an individual approach to the specific conditions and needs of a particular user [3,4]. Universal methodology, supporting design of integrated information acquisition systems, called MIAS (Manufacturing Information Acquisition System), enabling the systematic design of a production data acquisition system, aimed at company management support, has been proposed in [5]. The main factors hindering the development of a universal methodology for data acquisition production are a huge diversity of existing production systems associated with the variety of technological processes, differences in the scale of production, specificity and organizational culture of companies, as well as a multitude of IT solutions, supporting the different areas of a company. IT and control systems used in companies are often a patchwork of solutions from different periods of time, from different suppliers, that are frequently not compatible with one another. Issues of data acquisition from different production systems, including all stages (i.e. accessing raw data, preprocessing, archiving) and technical means (data sources, communication and interfacing) of data acquisition have been discussed in [6].

All previously mentioned factors are the cause, that data acquisition and integration of manufacturing systems, with a business area, should become the subject of research and development of new solutions that could improve the management of processes taking place in companies. For this purpose the Integrated Manufacturing Systems Laboratory in the New Technologies Centre of the Silesian University of Technology, was established and equipped with modern automation devices and advanced software, allowing research and development on the field of the acquisition of production data and integration of production systems with the business area of companies. It allows the conducting of research, and the development of control systems, as well as for lectures and workshops for students specialising in industrial automation, or production engineering and management.

\section{Concept of the Integrated Manufacturing Systems Laboratory}

The problem of the integration of the production system with the business area, being an important part of the Industry 4.0 concept [1] requiring full interconnectivity of machines, systems, processes and products in "smart" networks, can be divided into several specific tasks, such as:

- collecting data through sensors and other devices,

- acquisition of data from non-automated or partially automated production systems, requiring additional data sources, e.g. automatic identification systems,

- generating data in controllers (PLC - Programmable Logic Controller, CNC, etc.),

- supervisory control in HMI (Human-Machine Interface) and SCADA (Supervisory Control And Data Acquisition) systems,

- communication and interfacing in heterogeneous production system environments,

- data archiving and processing in both the middle-layer and business-layer systems (e.g. MES - Manufacturing Execution System, ERP - Enterprise Resource Planning). 
These subjects can be divided between two main areas: hardware (automated machines, actuators, semi-automatic data sources, control systems, hardware layer of communication interfaces) and software (standards, interfaces and formats of communication, industrial databases, MES systems and ERP software) [3].

In accordance with these objectives, a laboratory equipped with a set of hardware components, responsible for generating data (a small production system with sensors and automatic identification subsystems - vision system, barcodes and RFID - Radio-frequency Identification), industrial control and data processing systems (PLCs and other hardware), communication interfaces, as well as software (OPC - OLE for process control), $\mathrm{HMI} / \mathrm{SCADA}$, industrial databases, and Manufacturing Execution Systems and Enterprise Resources Planning) has been established.

\subsection{The specificity of communication in production systems}

All hardware and software elements of the Laboratory are connected by communication infrastructure, based on the Ethernet hardware layer. Ethernet network connects components that communicate using different protocols, including among others TCP/IP, Profinet, Modbus IP. Some elements are connected using other interfaces, such as RS-232, RS-485 (Modbus RTU), Profibus, CANbus and CC Link. There is also a set of modern IOLink devices (IO-Link masters, sensors and actuators) - IO-Link is a relatively new communication standard gaining popularity in European industry, allowing the creation of complex control systems with remote input/output islands, based on standard 3 or 4-wire cables, which allows the modernization of existing control system by modern sensors (smart sensors) and actuators [7]. The set of communication interfaces can be easily extended, allowing for testing different solutions used in companies.

The diversity of control and communication systems in the Laboratory allows the simulation of real situations commonly found in the industry - co-existence of hardware and software solutions from different periods of time and from different suppliers. Nowadays, communications is increasingly being dominated by solutions based on Ethernet and is compatible with international standards (e.g. Profinet), displacing older and proprietary communication solutions. Often, however, the older solutions (interfaces, operating systems), even over 20 years old are still used, and the company does not plan to abandon them, forcing integrators to create communication systems including those obsolete hardware components for a task of acquiring complete set of data on technological processes and equipment status [6].

\subsection{Acquisition of data from non-automated production systems}

Designers creating a production data acquisition system should not forget that usually not all parts of the production process are automated (automated parts of company operation are easier object of data acquisition, equipped with wide selection of data sources, requiring often only the establishment of appropriate interfaces), so in order to obtain a full picture of the situation, solutions allowing data acquisition from non-automated parts of the process should be developed as well [8].

In non-automated (or partially automated) production systems, there is a need to replace previously used manual methods of data acquisition (such as verbal communication and reports written in logbooks) with semi-automatic solutions, speeding up data acquisition and allowing for the immediate pre-validation. Such solutions may include automatic identification systems (barcodes, RFID), as well as methods based on image recognition systems, dedicated HMI stations with appropriate programs and other solutions, depending on the specifics of the processes subject to reporting [8]. Location of objects (products, lots 
of WIP, mobile machinery, means of transportation, etc.) and workers can be acquired using a relatively new class of systems - Real-Time Locating Systems (RTLs) [9].

In the Laboratory of Integrated Manufacturing Systems semi-automatic (or assistedmanual) data acquisition solutions are also represented [6], including 1D and 2D barcode technologies, RFID systems operating in different frequency bands, vision systems (advanced smart camera and vision sensors), as well as HMI touch panels, that can be programmed to support data acquisition tasks.

\subsection{Organization chart of the Laboratory}

Based on the above assumptions, the organizational structure of the Integrated Manufacturing Systems Laboratory, including a set of specialised workstations and additional hardware and software modules has been proposed (Figure 1).

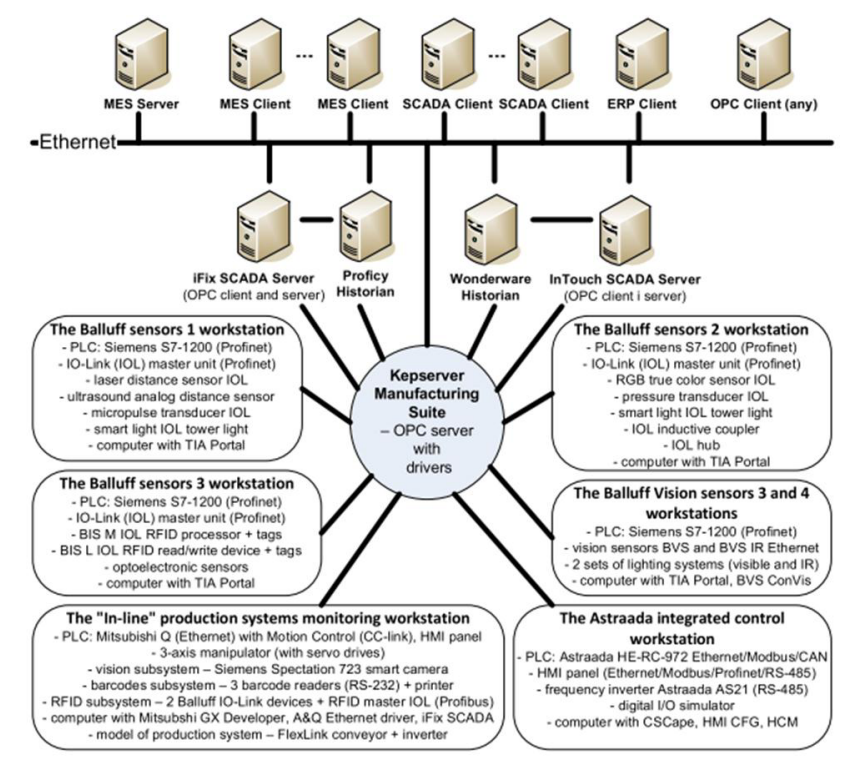

Fig. 1. Organization chart of the Integrated Manufacturing Systems Laboratory

Main workstations, subsystems and software modules of the Laboratory are as follows:

- The "In-line" production systems monitoring workstation

- The Balluff sensors 1workstation - IO-Link distance and position sensors

- The Balluff sensors 2 workstation - IO-Link pressure and colour sensors

- The Balluff sensors 3workstation - IO-Link RFID system, other various sensors

- The Balluff vision sensors 4 and 5 workstations - vision sensors and lighting

- The Astraada integrated control workstation - PLC, HMI, inverter, motor, sensors

- Software tools allowing programming of devices (PLCs, HMIs)

- The OPC server package, including drivers for various PLCs and devices (KEPServerEX Manufacturing Suite)

- SCADA systems (Proficy iFix, Wonderware InTouch, Citect SCADA)

- Industrial Databases (Proficy Historian, Wonderware Historian)

- MES software - (Proficy Plant Applications, Wonderware MES) 


\section{Main control and data acquisition workstations}

The Laboratory should provide opportunity to get acquainted with the widest range of hardware and software currently encountered in the industry. The workstations can be regarded as independent, allowing learning of the PLCs and I/O systems programming. They can also be considered as a part of an integrated system of data acquisition and supervisory control, acting as data sources and objects of supervisory control or management.

\subsection{The "In-line" production systems monitoring workstation}

It is the most complex and at the same time, the oldest workstation, forming the nucleus of the Laboratory, described previously in [10], partially rebuilt (exchange of TURCK RFID subsystem into Balluff IO-Link RFID system, the installation of various sensors and IOLink Smart Light programmable tower light). It is an integrated system that allows for the monitoring of the production process (the miniature modular production system to be monitored can be set inside, under the frame of the "In-line" workstation) with an advanced vision system (Siemens smart camera), RFID tags and bar codes, with the support of conventional sensors for industrial automation. The main function modules of the "In-line" workstation are three semi-automated data acquisition subsystems, a chassis acting as the supporting structure for the other elements (with 3-axis manipulator carrying Smart camera and one RFID heads), an advanced control system (based on Mitsubishi's Q series PLC and HMI panel), and mechatronic drives.

\subsection{The Balluff sensors 1, 2 and 3 IO-Link workstations}

These are 3 similar workstations built on the basis of Balluff's IO-Link elements and Siemens S7-1200 PLCs, where the emphasis is on the use of modern methods of communication between PLC and sensors / actuators. It has been decided to use a new, communication standard called IO-Link [7], currently gaining popularity, which allows two-way communication between the IO-Link master unit and slave devices - intelligent sensors (smart sensors) and actuators, using typical 3-wire cables. The IO-Link master unit can be connected to PLC or other higher-level device using Profinet, Profibus or other interfaces, which allows the easy creation of remote input/output islands. IO-Link is not regarded as an industrial network, because the I/O sockets of IO-Link master unit cannot be connected to the other master units on the other levels of the hierarchy (it is still possible to have more master units connected by Profinet or Profibus). IO-Link allows for the shortening of the distance between places where analogue data is collected and digitized A/D conversion can take place directly in smart sensors or in special analogue sockets of the IO-Link master, preventing distortion of the signal. Typically, it is possible to connect IO-Link smart sensors / actuators or standard discrete sensors to IO-Link master sockets and full diagnostics of master - sensors - actuators connections is also possible. Thanks to bi-directional communication, smart IO-link devices allow remote configuration and diagnostics without physical access to devices, which is very convenient when they are not easily accessible. IO-Link makes it easy to modernize the control system through the exchange of sensors and leaving the existing wiring in place. A diagram of the possible connections of devices in the IO-Link is shown in Figure 2. 


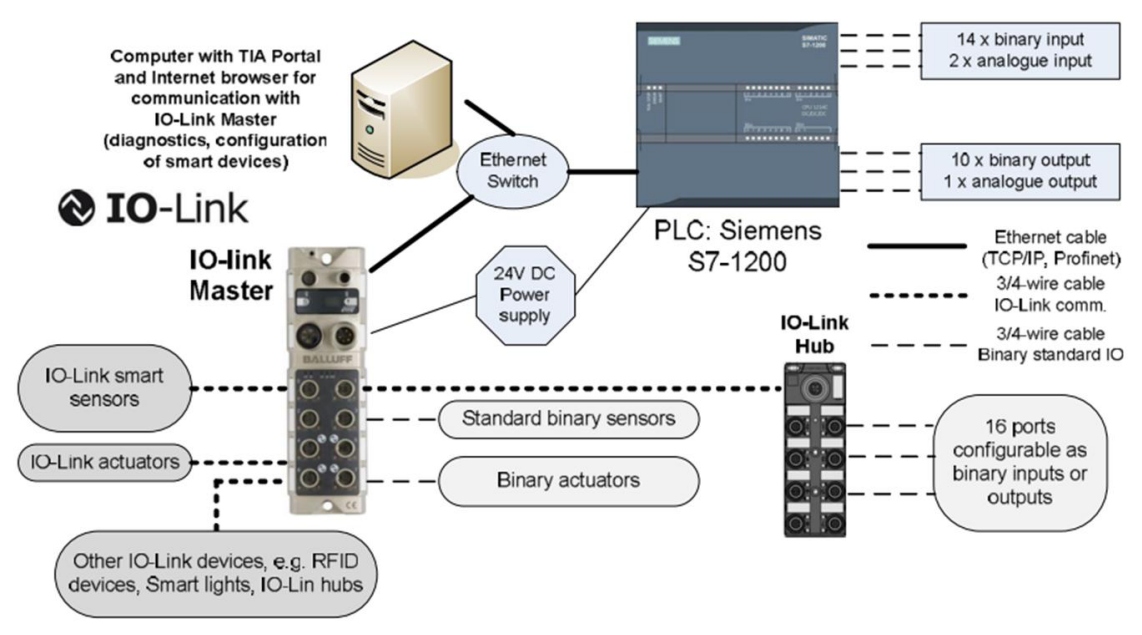

Fig. 2. Possible connections of IO-Link devices in the Laboratory's workstations

As mentioned before, these workstations are built on the basis of Siemens S7-1200 PLCs and Balluff's IO-Link master units, allowing for direct connection of up to 16 slave devices that communicate using the IO-Link protocol or standard discrete I/O signal. An interesting device is the IO-Link hub (installed in workstations 2 and 3), that allows the connection of up to 16 ( 2 pins in each of 8 sockets) standard discrete sensors or actuators (in this case it is not possible to connect smart sensors). Another interesting solution is the inductive coupler used in the Balluff sensors 2 workstation, allowing wireless transmission for a distance of up to $5 \mathrm{~mm}$ for both the IO-Link or SIO (Standard Input-Output) signal, as well as supplying power for the connected devices. This allows for easy communication with moving machine parts, replacing unreliable mechanical connections.

The other devices available in the Balluff sensors 1, 2 and 3 workstations are a variety of sensors (listed in Figure 1) and RFID devices, as well as output devices - Smart Light tower lights. The Balluff sensors 1 workstation, together with the specified devices is shown in Figure 3 (left). The workstation's main components are Simatic S7-1200 PLC (1) and IO-Link master unit BNI005H (2), allowing the connection of up to 8 intelligent IOLink sensors or actuators (connecting classic sensors is also possible), both interfaced using Profinet. PLC is equipped with a simulator and an ultrasound distance sensor (3), while remaining sensors, optoelectronic (laser) distance sensor BOD0012 (4) and Micropulse position sensor BTL1F5N (5) are connected using the IO-Link standard. The Smart Light BNI0086 (LED programmable tower light) (6) is currently the only output device (Figure 3, left).

\subsection{The Balluff vision sensors 4 and 5 workstations}

The main components of these workstations are Balluff's vision sensors (BVS 001M and BVS $001 \mathrm{H}$ ), the first of which requires illumination of the observed objects in visible light, and the second one requires infrared lighting), that differs from the conventional "full-size" or smart camera vision systems by the simplicity of its installation, application and programming, but they are slightly less capable (smaller resolution and configuration flexibility, the lack of a possibility to exchange lenses). It allows for $1 \mathrm{D} / 2 \mathrm{D}$ barcodes reading, feature presence/absence and inspection, pattern detection, position detection, dimension verification, edges counting, etc.. All configured inspections compensate for the parts rotation, minimizing the need to carefully position parts, while reducing setup costs [11]. The vision sensors communicate with the surroundings (Siemens PLC and a computer 
used for programming by ConVis configurator) via Ethernet and RS-232 interfaces. The outputs of the sensor signals can be discrete signal (success or failure of inspection), the numerical values of the parameters (the coordinates of objects, dimensions and distances), and strings read from the barcodes. An important element of the vision sensor workstations are sets of LED light sources, operating in the visible light or infrared - spotlight, linear and backlight. The vision sensors workstations enables research and teaching on the use of vision systems in production processes - the selection of suitable sensors, optical characteristics and lighting, adjusting the position of the sensor in the analyzed scene, configuration procedures, object recognition, as well as the programming of industrial controllers for use with vision sensors.

\subsection{The Astraada integrated control workstation}

The Astraada workstation (Figure 3, right) allows research and teaching on a number of issues related to the control of technological processes. The main elements are Astraada HE-RC972 PLC (7) with Ethernet (there are various protocols available, including Modbus IP, FTP and HTTP), RS232 and CAN interfaces, colour touch screen HMI (8) Astraada AS43TFT0725 (Ethernet, USB, 3xRS232/485 interfaces) and the Astraada AS21DRV20C4 frequency inverter (12), connected with the AC motor (13). The PLC communicates with the HMI panel via Modbus IP, and the HMI with a frequency inverter via RS485 (Modbus RTU), enabling visualization of the state and setting the parameters of the inverter. Inductive (10) and capacitive (11) sensors are also available, as well as an I/O simulator (9).
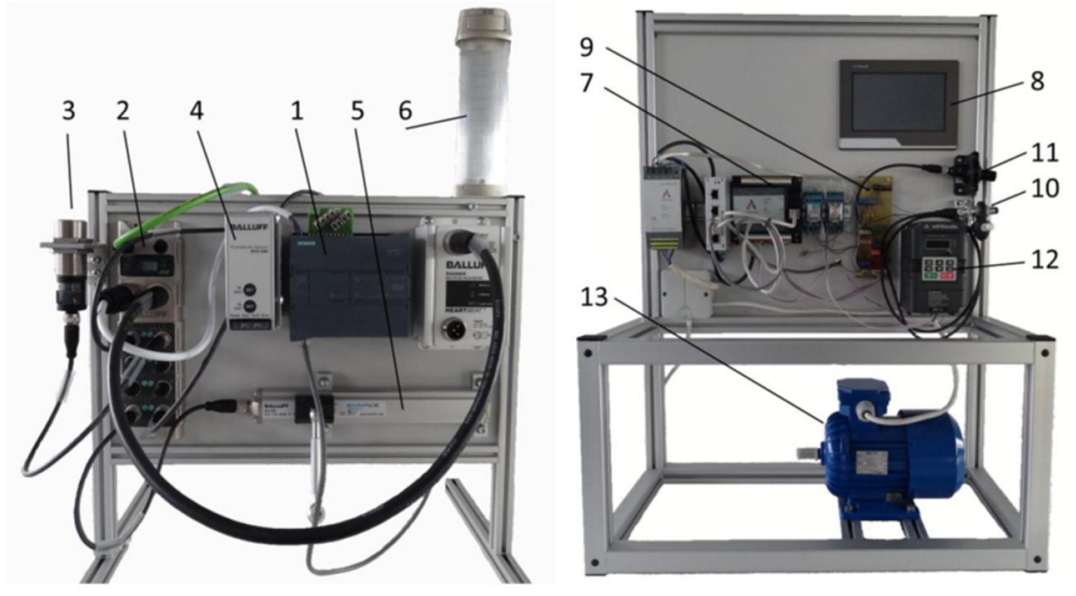

Fig. 3. The Balluff sensors 1 (left) and the Astraada integrated control (right) workstations

The range of possible exercises includes programming the controller, HMI and inverter and the cooperation of these devices using different media and protocols. The Astraada workstation is naturally integrated with the network of the Laboratory and higher level software, in particular KEPServerEX OPC server, that can communicate with the PLC and the HMI, allowing interaction with the hardware for any OPC client applications.

\section{Software used for the data acquisition and production systems integration}

The software installed in the Laboratory, combined with the hardware allows a complete representation of issues related to the Industry 4.0 concept, and thus the subject of 
integration of equipment, staff and circulation of materials in a thoughtful, orderly system, capable of delivering real-time information about the status of machines and equipment, execution of orders, crew activity, and product quality and genealogy. IP addresses of computers and other devices installed in the Laboratory belong to a common address pool, so all devices can communicate with each other, which makes PLC programming from any computer in the Laboratory or access to any data possible.

There are 3 main types of software installed in the Laboratory:

- software tools necessary for configuring and programming of devices (PLCs, HMIs, frequency inverters, vision sensors, IO-link masters and smart sensors),

- software interfacing between hardware and IT systems - OPC server and drivers, dedicated drivers,

- IT systems exchanging, archiving and consuming data from hardware sources - SCADA, Historian, MES, ERP, etc.

A study of the situation in the industry [12] shows that many of the problems with the integration of systems are associated with a variety of operating systems and security software used on computers, which resulted in the installation of different operating systems, anti-viruses and firewalls on the computers used in the Laboratory.

\subsection{The OPC server}

OPC was chosen as the main standard integrating hardware layer of the Laboratory workstations with SCADA, archiving and production management software due to its versatility, growing popularity and other advantages [13]. KEPServerEX OPC server has been selected as the main hardware-software interface. It is equipped with a wide range of drivers (KEPServer Manufacturing Suite) enabling communication with different devices. The OPC server communicates with devices from different manufacturers using dedicated drivers, and a variety of transmission media and protocols, while it shares the collected data in a standardized form (OPC protocol, SNMP, ODBC, XML, web services), allowing access to current and historical process data, alarms, for any compatible client software.

As a result, there is no need to create special communication drivers for each program that requires connection to specific hardware, moreover, the data is retrieved from the PLC to the OPC server only once, where it can be made available to many clients (reducing the communication load of PLCs). The OPC server can be the central point of integration of different areas of a company. OPC server can exchange data with other OPC servers, it is possible to apply redundant architecture, furthermore, some SCADA systems can be configured not only as an OPC client, but also an OPC server.

\subsection{HMI/SCADA, Historian and MES software}

The software installed in the Laboratory, communicating with the hardware by the OPC protocol includes: SCADA systems used for supervisory control and data acquisition from individual workstations, Historian industrial database useful for storing large amounts of data, and software belonging to higher level IT systems - production and business management level systems - Manufacturing Execution Systems (MES) and ERP. A common feature is the ability to use the OPC server as a source of data, although it is also possible to use dedicated drivers, and in the case of MES systems, the preferred data source is the Historian industrial database. The distinguishing features of the Historian, from typical SQL databases, include high performance of mass simple process data storage, ease of configuration, reliability, zero-downtime maintenance, automatic data compression, redundancy and the ability to pre-process the data (data integration - calculation of the synthetic data based on the values of many simple process variables) [14]. 
The Integrated Manufacturing Systems Laboratory uses integrated software packages, forming complete platforms, e.g. GE Intelligent Platforms (formerly Proficy) or Invensys Wonderware, which are complete solutions, including, inter alia, SCADA systems (GE iFix, Wonderware InTouch), industrial database (GE Historian, Wonderware Historian) and MES (GE Production Performance - formerly Proficy Plant Applications, Wonderware MES) [15]. This provides the opportunity to get acquainted with the solutions used in the largest enterprises [16].

\section{Example of the integration of workstations and IT systems}

The first task during the exemplary application was to get acquainted with the specifics of the communication protocols of existing equipment and checking the availability of OPC drivers. The next steps were: creating demonstration programs for each PLC and workstation, installing the appropriate OPC drivers in KEPServer, the configuring PLC OPC server connection, creating a database containing tags retrieved from the physical addresses in the PLCs, and validation of the communication using the OPC Quick Client application. The last stage of integration was gaining access to the data collected from the OPC client application. In this case HMI/SCADA iFIX was used, in which firstly the OPC communication driver had to be defined, then the database of tags was created, and finally SCADA applications using collected data from selected workstations were designed.

In order to provide data that can be accessed through OPC, demonstration programs have been developed for all workstation PLCs, using appropriate development environments (Mitsubishi GX IEC Developer, Simatic TIA Portal Step7, Cscape). These programs use various logic blocks, including Boolean operations, counters, timers, and other functions in order to generate different types of data, like Boolean, integer, real and strings, based on the state of inputs connected to simulators, sensors, or, in the case of the "in-line" workstation, data acquired from barcode and RFID readers.

\subsection{OPC server configuration and testing}

The main steps of setting up KEPServer OPC server are: adding of a channel (at this stage it is possible to choose which communication driver will be used), adding a device to the channel (defining device network address, protocol, communication settings and other parameters), and finally, adding tags (variables) to the devices.

Three separate channels have been defined because every workstation's PLC required another communication driver. The Mitsubishi Ethernet, Modbus TCP/IP Ethernet and Siemens TCP/IP Ethernet have been used, respectively, for workstations.

At the second stage PLCs have been added to the channels. Thanks to the local Ethernet network and its interfaces, all PLCs and PC with an OPC server in the same network segment, are available in uniformed TCP/IP address space, it is possible to exchange data with all PLCs and other networked devices (like IO-Link master units) in the Laboratory.

The most difficult part of the OPC set-up procedure is the defining of tags, because each PLC and protocol has its own methodology of addressing and accessing of different types of data, inputs, outputs, registers, variables and function blocks. In the case of relatively widespread Modbus protocol, it is possible to translate internal PLC addresses with the Modbus addressing translation table, that has to be combined with knowledge about the structure of a specific PLC (number of discrete/analogue inputs and outputs, flags, registers, system variables etc.). Mitsubishi and Siemens PLCs are both different and it is necessary to find technical data and addressing convention for each. KEPServer's help files describe the basics of addressing methods. The final effect of the OPC server configuration (Figure 
4) is a set of channels with defined devices and tag databases for each PLC device, accessing data changed in the PLC programs.

Validity of KEPServer configuration and communication can be verified using OPC Quick Client, available in the KEPServer set of programs. The status of communication with PLCs can be displayed, data quality on all defined tags should be "good", and value of variable in Quick Client should change together with changes in the PLCs.

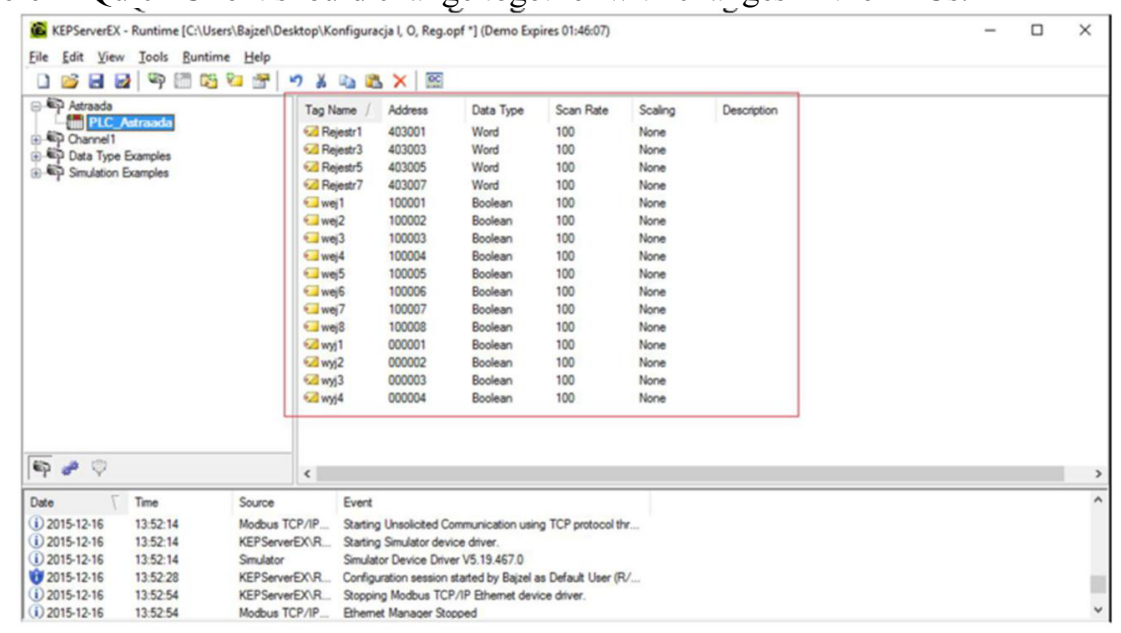

Fig. 4. KEPServer configuration for the Astraada workstation

\subsection{HMI/SCADA iFIX applications}

In order to check the last stage of integration, GE Intellution Proficy iFIX applications, displaying data acquired from workstations, have been developed. It required configuring the OPC driver in the iFIX configuration module, adding tags to the iFIX tag database, and then designing HMI screens displaying data from PLCs in various forms, like numerical display, bit lamps, character string displays, animated objects (Dynamo) and charts, etc.

\section{Conclusions}

The Integrated Manufacturing Systems Laboratory provides a unique opportunity to get acquainted and conduct exercises or research associated with the issue of the integration of manufacturing systems with software supporting company management at different levels. Previous solutions typically focused on one of these aspects, either the issue of designing, configuring and programming of automatic control systems, or only enterprise management support software (MES, ERP). Data processed in software supporting the management installed in the Laboratory are the actual, non-simulated data from workstations hardware, that can be configured and programmed for specific applications, making realistic presentation of these issues easier.

The main problems encountered during the operation of the Laboratory are the high degree of complexity and the variety of hardware and software solutions represented in it, which is a problem assuming the limited time of workshops, and existing conflicts between the presentation of issues of systems integration and programming of devices - presentation of issues of systems integration is possible if workstations are programmed with prepared demos, which often are overwritten during the PLC programming workshops.

The advantage of the OPC technology is that data acquired once from PLC can be reused many times in client applications. Experiments have shown that, thanks to the OPC, 
data can be obtained from different devices, but various difficulties have also been encountered. Access to simple data, such as the states of PLC's inputs, outputs and registers is relatively easy, but it is not always possible to obtain more complex data stored in function blocks specific for PLC manufacturers (e.g. to get data from optimized function blocks, dedicated Siemens software has to be used). Moreover, equipment from different producers and periods of time is often visible in the shopfloor - access to older devices, for which there is no documentation and source software can be a problem because of a lack of interfaces or knowledge on the meaning of data in the controller's memory - it has to be recovered through reverse engineering.

The Laboratory allows students to get familiar with the issues of integration of the various systems of control and management, very important in the context of the need of improving the efficiency of business management, and implementation of the Industry 4.0 concept.

The Integrated Manufacturing Systems Laboratory has been partially equipped thanks to cooperation from Balluff Sp. z o.o. and Balluff $\mathrm{GmbH}$.

\section{References}

1. S. Weyer, M. Schmitt, M. Ohmer, D. Gorecky, IFAC-PapersOnLine 48-3, 579 (2015)

2. K. Herbuś, P. Ociepka, IOP MSEng, 95, 012084, (2015)

3. A. Gwiazda, A. Sękala, Z. Monica, W. Banaś, Adv. Mat. Res., 1036, 1023 (2014)

4. A. Sękala, G. Ćwikła, G. Kost, IOP MSEng, 95, 012097 (2015)

5. G. Ćwikła, AppMechMat, 474, 27 (2014)

6. G. Ćwikła, AdvMatRes, 837, 618 (2014)

7. http://www.io-link.com/share/Downloads/At-a-glance/IO-

Link_System_Description_engl_2013.pdf (2013)

8. G. Ćwikła, D. Krenczyk, A. Kampa, G. Gołda, IOP MatScEng, 95, 012153, (2015)

9. N. Pirzada, M. Yunus Nayan, F. Subhanc, M. Fadzil Hassan, M.A. Khan, AASRI Procedia 5, 92 (2013)

10. G. Ćwikła, Adv. Mat. Res., 837, 334 (2014)

11. http://www.balluff-ua.com/pdf/bvs_manual_en.pdf(2015)

12. L. Monostori, Procedia CIRP 17, 9 ( 2014 )

13. G.G. Florea, L.A. Ochean, ProcIFAC InfContrProbManuf,1844 (2012)

14. B.R. Mehta, Y.J. Reddy, Industrial Process Automation Systems. Design and Implementation (Elsevier, 2015)

15. M. Bączkowicz, A. Gwiazda, IOP MatSciEng, 95 (2015)

16. K. Herbuś, G. Kost, D. Reclik, J. Świder, AdvMatRes, 837, 582 (2014) 